\title{
Patent Portfolio Analysis Related to Advanced VR Technologies and Applications for Treating Driving Phobia
}

\author{
A.J.C. TRAPPEY ${ }^{\mathrm{a}, 1}$, C.V. TRAPPEY ${ }^{\mathrm{b}}$, R.R.T. KUO ${ }^{\mathrm{c}, 2}$, A.P.C. LIN $^{\mathrm{d}}$ \\ a, c, d Department of Industrial Engineering and Engineering Management, National \\ Tsing Hua University, Hsinchu, Taiwan \\ ${ }^{b}$ Department of Management Science, National Yang Ming Chiao Tung University, \\ Hsinchu, Taiwan
}

\begin{abstract}
Virtual reality technology enables people to interact with objects in threedimensional simulated environments. The technology has been applied in many fields such as entertainment, education, and healthcare. Health treatment applications include psycho therapeutical treatments for post-traumatic stress disorders related to driving phobias. Patents that have been filed for treating patients diagnosed with driving phobias are searched and collected using global patent platforms including Derwent Innovation's solution. Patent analytic methods, including clustering, key term frequency analysis, correlation matrix creation, Latent Dirichlet Allocation (LDA), and technology function matrix mapping, are applied to analyze the patent portfolio and identify key technology development trends. The research goal is to identify patent strategies related to driving-related virtual reality psychotherapy treatment. The research identifies six technology clusters, including virtual reality technologies, vehicle driving simulations, diagnosis of driving phobia through bio-signal analysis, and artificial intelligence for health systems. Based on the cluster analysis, virtual reality related patents are increasing over the last decade. A lesser number of patents focus on virtual reality technology that includes exposure therapy for driving phobia treatment. The patent mining results show that virtual reality exposure therapy for driving phobias is still in the growth stage. There is a research gap for new treatments for driving phobias and other phobias such as post-traumatic stress disorder, which have great potential for transdisciplinary engineering research.
\end{abstract}

Keywords. Virtual reality exposure therapy, driving phobia, post-traumatic stress disorder, patent portfolio, vehicle simulation

\section{Introduction}

Virtual reality (VR) is an interactive and immersive experience where people interact within a lifelike environment created by integrating computer components. Driving simulation is a popular VR application where users equipped with VR apparatus experience driving a car in a virtual environment. The major advantage of VR driving simulation is safety. Users in a virtual environment do not expose themselves to actual road conditions yet the driving experience is very realistic and approaches the experience of actually driving a car. The virtual environment might be overwhelmingly realistic without being life-threatening. Researchers are developing VR driving programs and incorporating this technology into psychotherapy for driving phobia treatment since the

${ }^{1}$ Corresponding author: Professor Amy Trappey, email: trappey@ie.nthu.edu.tw.

${ }^{2}$ Corresponding author: Routine Kuo, email: s108034518@m108.nthu.edu.tw 
patient is not exposed to the physical dangers of losing control of a motor vehicle on an actual road and causing harm to patient, the therapist, or others.

Driving phobia is a type of post-traumatic stress disorder (PTSD) associated with drivers who experience serious traffic accidents or develop unrealistic fears of driving through traumatic experiences associated with driving, such as the loss of a spouse who died in an accident present at the time. People may be severely impaired in their daily lives when suffering from driving phobia since it may limit employment and the ability to travel long distances from home. An emerging method to treat driving phobia uses VR technology. This medical approach is called Virtual Reality Exposure Therapy (VRET) and is a transdisciplinary engineering research topic. The research combines virtual reality immersive-based technology and psychotherapy. Using VRET, patients are allowed to expose themselves to a simulation of the phobic surroundings but do not physically encounter the feared situations. The immersive exposure conditions can be adjusted through patient feedback to protect patients from excessive mental stress that may worsen the mental condition. Patients are gradually exposed to fearful driving conditions that they can tolerate inside the immersive environment. Through adaptive exposure, the patient increases self-confidence in driving, and with continued therapy in real-world conditions will be able to drive without fear.

This research focuses on patents related to VRET driving phobia and VR driving applications. Studying related patents provides a concise view of the new technologies applied in the industry. Understanding the opportunities for creating patent portfolios and finding potential opportunities for new technologies in the VRET domain provides a strategic advantage. This research is divided into the literature review, the research methodology, patent portfolio analysis, and the conclusions. The literature review analyses non-patent peer-reviewed literature on VRET and the VR driving domain. In the methodology section, patent search strategies are devised to collect patents from the Derwent Innovation database, and analytic tools, including clustering (k-means), NTFIDF, Latent Dirichlet Allocation (LDA), and the technology function matrix (TFM) are used to analyze patent topics for and classification. An ontology is constructed to present the analytical results. The patent portfolio analysis describes strategies applied to VRET and VR driving to create new development opportunities. The conclusions of this research are summarized in Section four.

\section{Literature Review}

VRET is a virtual reality system that assists the doctor and patient by integrating exposure and data collection in a safer clinical setting. By combining virtual reality devices and biosignal sensors, clinicians can customize and control the scene and simulate a virtual environment in a three-dimensional space to provide patients with a better sense of presence, provide adaptive exposure to increase exposure effectiveness and safety. VRET treatment can be provided in a small clinical space providing patient privacy (North, North \& Coble, 1997). The clinician can control the environmental characteristics that lead to patient embarrassment, complexity, and irritation. The treatment is highly scalable, and the treatment environment's controllability demonstrates the flexibility of the system (Romano, 2005).

Several technology companies have invested resources in the development of virtual reality systems and components. Facebook acquired Oculus and released Rift which is a mobile version of Go and nowQuest. Google created Daydream for augmented reality solutions. HTC Vive, Sony Playstation VR, and Apple are developing virtual reality 
devices (Miloff, et al., 2020). With increasingly powerful hardware development resources, a solid foundation has been laid for advanced development of VRET used in clinical settings.

Literature related to VRET driving phobia treatment has been studied. In one VRET driving phobia pre-test experiment conducted at the Taiwan National Tsing Hua University (NTHU), 31 subjects participated in a seven-level driving scenario. The clinical design used a pre-test analysis to identify students with driving anxiety, a control group and random assignment. The exposure simulated driving on a country road, a highway, and a mountain road during the day and at night. The level of fear inducing driving exposure increases with successful completion of lower levels of exposure. The heart rate, respiration rate, skin conductance, and body temperature data were collected to measure the degree of anxiety degree. The pre-test result examined the effectiveness of the VRET system. The subject's anxiety increases with level progression, verifying that the VRET system design affects the subjects' mental state (Trappey, et al., 2020). Some issues were identified in pre-test experiment, and refinements were made in a new clinical experiment. In the refinement experiment, 130 subjects filled out the pretreatment questionnaire. Sixty students with high fear of driving were randomly placed on a waiting list or selected as the treatment group to participate in clinical VRET experiment. The experiment results showed a significant improvement for the treatment group and their fear of driving in the exposure environments was reduced. The subjects rated that the immersive experience of the refinement experiment more favorably than subjects in the initial pre-test experiment (Trappey, et al., 2021). In another study (Wald $\&$ Taylor, 2000), patients received three treatments within ten days. The treatment included four VR driving scenarios. As a result, the patient's anxiety was reduced. The anxiety and avoidance scores began to decline after treatment and were maintained after a seven-month follow-up study.

Virtual reality technology has been applied to driver training. For professional driver research (Gąsiorek, et al. 2019), an experiment was used to compare the driver training effects between 2D and 3D simulations. Three dimensional simulations provide a more realistic immersive environment and realistic driving conditions. The drawback was some subjects could not endure the dizziness of $3 \mathrm{~d}$ motion sickness caused by $3 \mathrm{D}$ simulators and preferred 2D simulations. A study by Fitzpatrick and Mangalore (2019) evaluated the use of virtual reality headsets to measure driving performance for driving simulation and training. The study provides justification for the use of VR headsets to better understand a drivers' hazard anticipation behavior. The results were used to create a risk awareness training program. Physiological data were collected in the design of a virtual reality system (Herumurti, Yuniarti, et al., 2019). Herumurt's study proposed to treat a patients' public speaking anxiety. The study simulates the scene of a speech on stage and the audience off stage. The system measures the subject's heart rate to determine changes given the difficulty of exposure level and different audience responses. If the patient's heart rate is very high, the researchers hypothesized the speaker was very anxious, and the simulation was changed so the audience would doze off and be inattentive, making the subject less anxious. If the subject's heart rate was not high, the system would simulate an audience of attentive listeners and increase the difficulty of the speech to be delivered. Studies have confirmed that blood oxygen is the least effective among the many physiological feedback for measuring emotions, while blood volume and skin response are the most effective (Trivedi, 2018). A study recruited 36 people with fear of flying and 22 people without to participate in a VRET experiment. During the treatment, the participants' heart rate, skin resistance, and skin temperature 
were used as indicators to record the physiological trends. As a result, the fearers and non-fearers show a significant difference in skin resistance, and as the treatment progressed, the skin resistance of the fearers tend to approach the non-fearers' skin resistance, showing that the skin resistance can effectively reflect human anxiety (Wiederhold, et al., 2002). Some studies don't directly define emotions and classify the exposure as going well or going badly, and then adjust the level of difficulty over time using this classification (Dingli \& Bondin, 2019). A novel virtual reality driving system to teach driving skills to adolescents with autism spectrum disorder was introduced by Zhang, et al. (2017). This driving system monitors eye movement, electroencephalography, and peripheral physiology data in addition to driving performance data. The objective of the paper is to integrate multimodal information to measure cognitive load during driving such that driving tasks can be individualized for optimal skill learning. Results indicate that multimodal information can be used to measure cognitive load with high accuracy.

The concepts of convolution and machine learning have been applied to physiological data analysis. In Jacob Kritikos's research, he used skin resistance to measure emotion and used continuous deconvolution analysis to disassemble data to optimize the analysis results (Kritikos, et al., 2019). Another study used a 3x3 matrix to define the level of arousal, dividing the physiological data every 10 seconds as input for machine learning classification. The research results show that feature extraction is a key step in the classification process and support vector machines improve sentiment classification accuracy (Hinkle, et al., 2019).

To enhance the VRET system, it is important to improve the sense of presence for users when immersed in the virtual environment. Sheridan (2000) claims that four factors affect the sense of presence and include information quantity, sensor position and orientation, change of relative location of objects, and the active imagination in suppressing disbelief. Once these factors are managed, the VR will become more authentic and improve the user's immersion in the virtual environment. Illusion of presence also strengthens the users' sense of presence, which refers to a psychological phenomenon known as embodiment (Waldrop, 2017). Slater, et al. (2010) discovered that when the subject's virtual body is threatened with harm, the heart rate shifts into fright mode.

Heyse, et al. (2019) provide a semantic ontology for representing relevant concepts and relations related to VR anxiety therapy. The concepts used in their exposure therapy and VRET ontologies are refined using our research methods.

\section{Research Methodology}

In this section, patents related to VRET and VR driving domains are collected using Derwent Innovation search database. It is a proprietary patent research application that provides trusted patent data from patent offices worldwide. The patent search strategies are presented in section 2.1; clustering (k-means), NTF-IDF, and LDA are applied as patent classification tools to better understand the most critical VRET and VR driving domains Sections 2.2 and 2.3. The technology function matrix (TFM) for patent portfolio strategic analysis is described in Section 2.4. Using the patent classification results, the VR driving ontology is depicted as a tree diagram in Section 2.5. 


\subsection{Patent search strategy}

This research collects patent data from Derwent Innovation and the results are presented in Table 1. The keywords combinations, the IPCs, and yearly filter setting used as patent search strategies have been listed. A total of 428 related patents are collected and analyzed.

Table 1. Patent search strategy.

\begin{tabular}{llr}
\hline \multicolumn{3}{c}{ Virtual reality immersive technology \& driving phobia } \\
\hline Keywords combinations & Virtual reality + Driving phobia & (195 outcomes) \\
& Virtual reality + Driving training + Therapeutic & (50 outcomes) \\
& Virtual reality + Virtual vehicle + Artificial intelligence (54 outcomes) \\
& Virtual reality + Driving simulation + Sensor & (132 outcomes) \\
& Virtual reality + Psychotherapy + Car accident & (15 outcomes) \\
\hline IPCs & G06F, G06T, G06K, G06Q, G09B, G02B, G16H, A61B, A63F \\
\hline Application year & 1999 2020 & \\
\hline
\end{tabular}

This study uses text mining to cluster and analyze the patents. The following uses kmeans, LDA, and NTF-IDF methods to discuss the patent analytics.

\subsection{Clustering}

$\mathrm{K}$-means is a clustering method used to partition a set of data with similar features into $\mathrm{k}$ groups (MacQueen, 1967). It has been successfully applied in patent cluster analysis in our previous research (Hsu, et al., 2006), so we adopt k-means in this patent research to better understand the most important topics in the VRET domain. 428 related patents were classified into 13 clusters, and the NTF-IDF algorithm is applied after clustering to rank the frequency of words in each cluster. If different clusters have similar word frequency, these clusters are integrated into a single cluster. The 13 clusters are reduced to 7 clusters as presented in Table 2. These clusters define the main topics related to VRET and the VR driving domain.

Table 2. Seven clusters classified using k-means and NTF-IDF.

\begin{tabular}{ll}
\hline \multicolumn{1}{c}{ Cluster } & \multicolumn{1}{c}{ Meaning } \\
\hline System information & $\begin{array}{l}\text { The internal information flow of the software used when running } \\
\text { the VR system }\end{array}$ \\
\hline VR scene construction & Design and build VR scene \\
\hline Subject's feedback & The sensor receives the user's physical feedback \\
\hline Visual & The screen presented to the user in the headset \\
\hline Vehicle simulation & $\begin{array}{l}\text { Increasing the authenticity of VR driving situations, and its key } \\
\text { terms include simulation, drive, game, and motion }\end{array}$ \\
\hline VR training & Applications related to training \\
\hline Apparatus \& System & The hard drives and device that will be used \\
\hline
\end{tabular}

\subsection{Latent Dirichlet Allocation}

LDA is a generative probabilistic model applied to collections of discrete data such as text corpora to identify topics, which has been successfully applied in our previous solar power patent research published in Applied Sciences (Trappey, et al., 2019). In this research, LDA is applied to classify the topics among patents and identify the key terms in each topic. A total of seven subtopics can be merged into four main topics: sensory receptor, simulation, scenario design, and application, as shown in Table 3. 
Table 3. Four main topics classified using Latent Dirichlet Allocation.

\begin{tabular}{ll}
\hline Main topics & Meaning \\
\hline Sensory receptor & Hardware receives user information \\
\hline Simulation & Design and construction of virtual environment \\
\hline Scenario design & Sensory stimulation from the device to the user \\
\hline Application & VR driving applications, such as gaming, training, and healthcare \\
\hline
\end{tabular}

\subsection{Technology function matrix}

The Technology Function Matrix (TFM) is a useful approach for patent analysis. The matrix organizes information from patents so that users can readily visualize and interpret quantitative information that maps patented technologies to related functions. The map provides a brief sketch of patent development trends within the given domain, helping investigators understand what technologies and functions are leading industry trends and the gaps in development that offer opportunities (Jhuang, et al., 2017). This research analyzed and interpreted the patent search results using the Themescape Map function provided by Derwent Innovation. The function identified six leading technologies, including vehicle simulation, virtual reality technology, PTSD diagnosis, bio-signal analysis, health systems, and artificial intelligence (self-adaptive systems). To define the functions, three applications related to VR driving were defined based on LDA results: entertainment, driving training, and healthcare.

A total of 428 patents related to VR driving were analyzed using the TFM, and the output result is presented in Table 4. The two most popular fields are vehicle simulation (T01) and VR technology (T02) mapped with the function driving training (F03). Our research focuses on the development of VRET for driving phobia, which is highly related to PTSD diagnose (T03) with VRET (F02) and PTSD diagnose (T03) with driving training (F03). Based on TFM findings, patents related to VRET function are relatively scarce, with approximately one-third of patents related to driving training function. There is a noticeable gap and opportunity to develop VRET for driving phobia applications.

Table 4. Technology function matrix.

\begin{tabular}{|c|c|c|c|c|c|c|}
\hline & & & \multicolumn{4}{|c|}{ Function } \\
\hline & & & F01 & F02 & F03 & F04 \\
\hline & & & Entertainment & VRET & $\begin{array}{l}\text { Driving } \\
\text { training }\end{array}$ & $\begin{array}{l}\text { Healthcare } \\
\text { application }\end{array}$ \\
\hline \multirow{7}{*}{$\begin{array}{l}\overrightarrow{0} \\
\overbrace{0}^{0} \\
0 \\
0 \\
0 \\
0 \\
0\end{array}$} & T01 & Driving simulation & 14 & 6 & 92 & 12 \\
\hline & T02 & VR technology & 42 & 61 & 178 & 26 \\
\hline & T03 & PTSD diagnoses & 1 & 18 & 10 & 8 \\
\hline & T04 & Bio-signal analysis & 7 & 16 & 33 & 26 \\
\hline & T05 & Health system & 3 & 5 & 8 & 23 \\
\hline & T06 & Artificial intelligence & 7 & 15 & 39 & 5 \\
\hline & & Total & 74 & 121 & 360 & 100 \\
\hline
\end{tabular}

\subsection{Ontology design}

The ontology was presented using a tree diagram. The root node is the main topic of VR driving. The nodes one step lower than the root node in the hierarchy are the four sub- 
domains, which are the main topics identified via LDA (sensory receptor, simulation, scenario design, and application). Each sub-domain is further sub-classified into detailed technologies and applications. The ontology is presented in Figure 1.

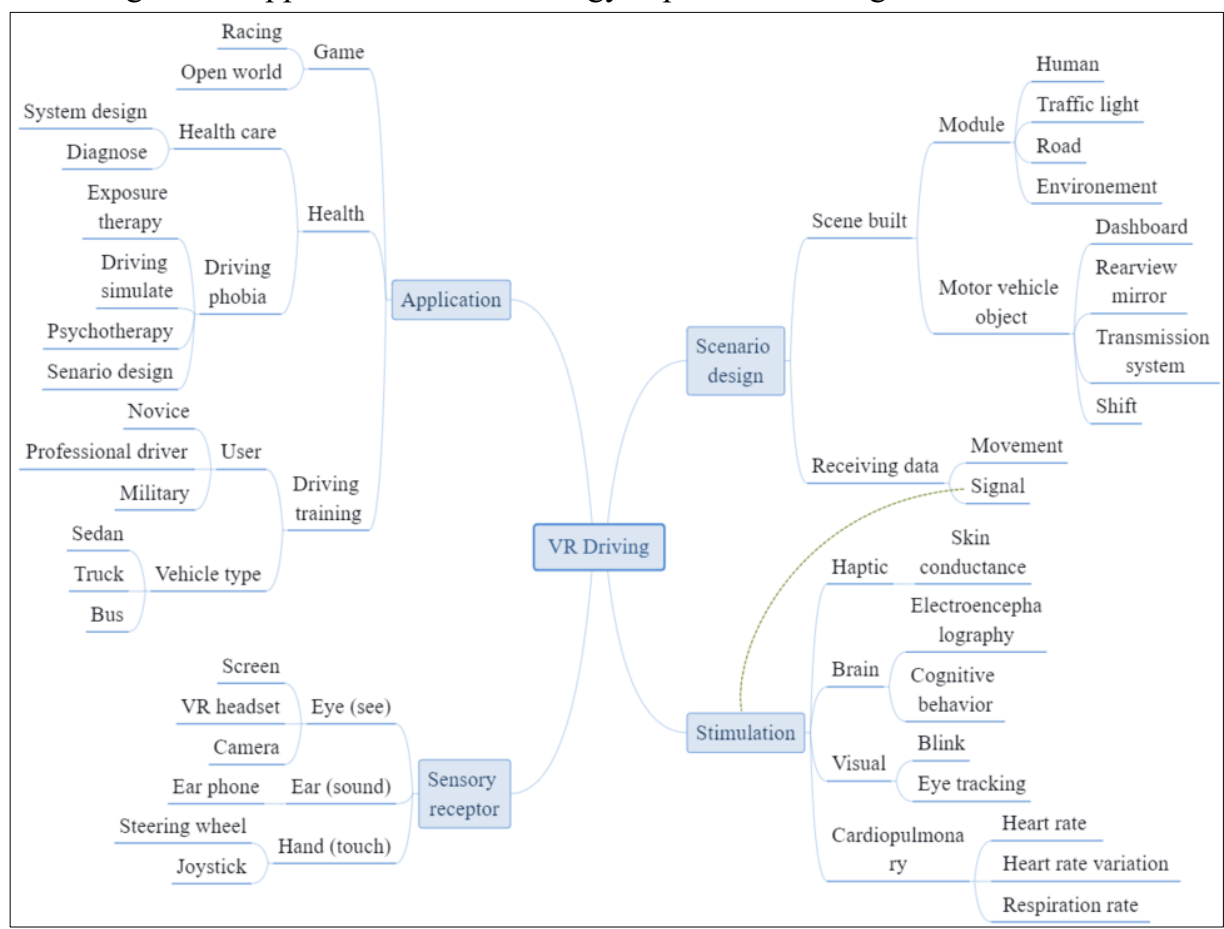

Figure 1. VR driving ontology design.

The first sub-domain is the sensory receptor. Subjects receive stimulations through sensory receptors - eye (visual), ear (sound), and hand (touch). The second sub-domain is stimulation feedbacks. When subjects were receiving stimulations, they will have haptic, brain, visual, and cardiopulmonary feedback. These stimulation feedbacks are often used to evaluate subjects' physiological status while using VR driving programs. The third sub-domain is scenario design. The sensory stimulation given to the user mostly depends on scene built and game objects, including interactive avatars, traffic lights, road, and background environment. The fourth sub-domain is the application that VR could be applied in gaming (entertainment), healthcare, and driving training. VR gaming has exploded in popularity in recent years with VR equipment become popular. People have always been concerned with health issues, and VR technology is suitable for diagnosing and treating mental disorders. The most widely used field of VR driving technology is driving training - regardless a novice driver wants to experience a drive on the road, veterans want to learn how to drive a truck, or even military training purposes. Various kinds of VR programs have been designed for driving training.

\section{Patent portfolio analysis}

Six technologies and four functions that correspond to the research domain were identified using TFM. Further information about the patent portfolio strategies used in different countries is summarized. Table 5 and 6 are the patent search results of each technology and function along with the number of related patents registered in the 
country patent platforms: USPTO (US), CNIPA (China), EPO (Europe), JPO (Japan), KIPO (Korea), and TIPO (Taiwan).

Assignees in the U.S. emphasize PTSD diagnosis (T03), health systems (T05), driver training (F03), and healthcare applications (F04). The results indicate that psychological medical technology in the US is more mature in comparison to other countries.

Assignees in China focus on vehicle simulation (T01), virtual reality technology (T02), artificial intelligence (T06), entertainment (F01), and VRET (F02). These technologies belong to emerging fields, so assignees in China pursue opportunities that have high potential strategic benefits.

Assignees in Japan are focused on driving simulation (T01). The automotive industry in Japan plays a leading role globally, so it is reasonable for Japanese assignees to focus on the automotive industry. For example, Toyota, Epson, Honda, and Hitachi automotive are the top Japanese assignees in this field. Assignees in Korea focus on bio-signal analysis (T04). The top assignees in this field are Samsung Electronics, LG Electronics, and Korea Electronics Technology.

The number of patents in Taiwan is fewer than other countries. Assignees in Taiwan often file their patent applications in other countries such as U.S., Japan, Europe, Korea, and China, because Taiwan is not a member of the World Intellectual Property Organization, and the market share in Taiwan is smaller than other countries.

T02 is Virtual reality technology and F02 is VRET. The number of patent applicants in T02 is approximately 100 times greater than patent applicants in F02. In comparison to other functions (entertainment, driving training, healthcare applications), there are relatively few patents related to VRET. Although people have studied about few companies and researchers have applied VR technology to exposure therapy and phobia treatment, our research domain is strategically important and in the introduction stage of development with few patents to challenge new developments.

Table 5 and Table 6 show relatively large markets for VRET related patents in Europe, Japan, and Korea. There have been many patents in the U.S. and China, which means that this technology may be relatively mature in these two regions, and if someone invests in it again, they may encounter patent landmines. As for Taiwan, although it looks like a blank market, its market share may be too small, making the investment efficiency not high. On the contrary, Europe, Japan, and Korea have a certain number of patents, which means they have a certain degree of maturity and investability, but they are not as easy to step on landmines as the U.S. and China, so they are suitable regions for applying for VRET-related patents.

Table 5. Technology related patents registered in different countries.

\begin{tabular}{lcccccc}
\hline & $\begin{array}{c}\text { Vehicle } \\
\text { simulation }\end{array}$ & $\begin{array}{c}\text { VR } \\
\text { technology }\end{array}$ & $\begin{array}{c}\text { PTSD } \\
\text { diagnoses }\end{array}$ & $\begin{array}{c}\text { Bio-signal } \\
\text { analyze }\end{array}$ & $\begin{array}{c}\text { Health } \\
\text { system }\end{array}$ & $\begin{array}{c}\text { Artificial } \\
\text { intelligence }\end{array}$ \\
\hline USPTO (US) & 7540 & 12267 & 350 & 628 & 4213 & 5947 \\
\hline CNIPA (China) & 23867 & 17530 & 51 & 245 & 2200 & 12452 \\
\hline EPO (Europe) & 3319 & 3105 & 111 & 243 & 1201 & 900 \\
\hline JPO (Japan) & 6681 & 1986 & 99 & 406 & 518 & 1052 \\
\hline KIPO (Korea) & 2273 & 4501 & 52 & 2095 & 345 & 3316 \\
\hline TIPO (Taiwan) & 224 & 887 & 12 & 35 & 160 & 179 \\
\hline
\end{tabular}

Table 6. Function related patents registered in different countries.

\begin{tabular}{lcccc}
\hline & Entertainment & VRET & Driver training & $\begin{array}{c}\text { Healthcare } \\
\text { applications }\end{array}$ \\
\hline USPTO (US) & 4745 & 206 & 10297 & 5648 \\
\hline
\end{tabular}




\begin{tabular}{lcccc}
\hline CNIPA (China) & 6222 & 362 & 5977 & 3395 \\
\hline EPO (Europe) & 1162 & 60 & 6289 & 1533 \\
\hline JPO (Japan) & 1074 & 57 & 2388 & 870 \\
\hline KIPO (Korea) & 716 & 97 & 1123 & 488 \\
\hline TIPO (Taiwan) & 353 & 10 & 144 & 163 \\
\hline
\end{tabular}

\section{Conclusion and contribution}

This research studies the transdisciplinary engineering applications of virtual reality immersive technology and psychotherapy. The immersive technology of virtual reality driving is in the early stage of development. The text-mining results of clustering (kmeans) and LDA have classified the key terms and main topics of VR driving-related patents, helping identify the core technologies in this domain. Although there are VR driving applications for entertainment, driver training, and healthcare, few patents are related to driving-related medical treatments or VRET. From patent portfolio analysis, we noticed that most of the VRET patent research comes from the patent offices of the U.S. and China, while it has more opportunities to apply VRET related patents in Europe, Japan, and Korea.Further, for advanced VR technologies and AI technologies that can be largely applied to VRET, we found the patent counts in VRET functional categories (F02) are much smaller than their driving training (F03) applications. The result indicates a research gap for new treatments for driving phobias and other phobias, but it also represents great potential for transdisciplinary engineering applications of virtual reality immersive technology and driving phobia treatment.

\section{Acknowledgement}

The research is partially supported by the research grants of Ministry of Science and Technology, Taiwan (Grant numbers: MOST-108-443 2221-E-007-075-MY3 and MOST-108-2410-H-009-025-MY2).

\section{References}

[1] M. M. North, S. M. North, J. R. Coble, Virtual reality therapy: an effective treatment for psychological disorders, Studies in health technology and informatics, 1997, pp. 59-70.

[2] D. M. Romano, Virtual reality therapy, Developmental medicine and child neurology, 2005, Vol. 47(9), pp. 580 .

[3] A. Miloff, P. Lindner, P. Carlbring, The future of virtual reality therapy for phobias: Beyond simple exposures, Clinical Psychology in Europe, 2020, Vol. 2(2), e2913.

[4] A.J.C. Trappey, C.V. Trappey, C. M. Chang, R. R Kuo, A. P. Lin, C. H. Nieh, Virtual Reality Exposure Therapy for Driving Phobia Disorder: System Design and Development, Applied Sciences, 2020, Vol. 10,4860

[5] A.J.C. Trappey, C.V. Trappey, C. M. Chang, M. C. Tsai, R. R Kuo, A. P. Lin, Virtual Reality Exposure Therapy for Driving Phobia Disorder (2): System Refinement and Verification, Applied Sciences, vol. 11(1), 2021, 347.

[6] J. Wald, S. Taylor, Efficacy of virtual reality exposure therapy to treat driving phobia: a case report, Journal of behavior therapy and experimental psychiatry, 2000, Vol. 31(3-4), pp. 249-257.

[7] K. Gąsiorek, E. Odachowska, A. Matysiak, M. Pędzierska, Virtual Reality Technologies in the Training of Professional Drivers. Comparison of the 2D and 3D Simulation Application, International Scientific Conference Transport of the 21st Century. Springer, Cham, 2019, pp. 133-142.

[8] C. Fitzpatrick, G. P. Mangalore, S. Samuel, M. Knodler Jr, Y. Ebadi, D. Fisher, Risk Awareness and Perception Training using VR Headsets: The Validation of VR Headsets to Measure Hazard Anticipation Behaviors, University of Massachusetts, Amherst, 2019.

[9] D. Herumurti, A. Yuniarti, P. Rimawan, A. A. Yunanto, Overcoming glossophobia based on virtual reality and heart rate sensors, IEEE International Conference on Industry 4.0, Artificial Intelligence, and Communications Technology (IAICT), Bali, 2019, pp. 139-144. 
[10] P. G. Trivedi, Human Emotion Recognition from Physiological Biosignals (Unpublished thesis), Texas State University, 2018.

[11] B. K. Wiederhold, D. P. Jang, S. I. Kim, M. D., Physiological monitoring as an objective tool in virtual reality, Cyberpsychol Behav., 2002, Vol. 5(1), pp. 77-82.

[12] A. Dingli, \& L. Bondin, Realtime Adaptive Virtual Reality for Pain Reduction, IEEE Conference on Games (CoG), London, 2019, pp. 1-4.

[13] L. Zhang, J. Wade, D. Bian, J. Fan, A. Swanson, A. Weitlauf, Z. Warren, N. Sarkar, Cognitive load measurement in a virtual reality-based driving system for autism intervention, IEEE transactions on affective computing, 2017, Vol. 8, pp. 176-189.

[14] J. Kritikos, G. Tzannetos, C. Zoitaki, S. Poulopoulou, D. Koutsouris, Anxiety detection from Electrodermal Activity Sensor with movement \& interaction during Virtual Reality Simulation, The 9th International IEEE/EMBS Conference on Neural Engineering (NER), San Francisco, 2019, pp. 571576.

[15] L. Hinkle, K. Khoshhal, V. Metsis, Physiological Measurement for Emotion Recognition in Virtual Reality, The 2nd International Conference on Data Intelligence and Security (ICDIS), South Padre Island, 2019, pp. 136-143.

[16] T. Sheridan, Interaction, imagination and immersion some research needs, Proceedings of the ACM symposium on Virtual reality software and technology, Seoul, 2000, pp. 1-7.

[17] M. M. Waldrop, News feature: Virtual reality therapy set for a real renaissance, Proceedings of the National Academy of Sciences, 2017, Vol. 114(39), pp. 10295-10299.

[18] M. Slater, B. Spanlang, M.V. Sanchez-Vives, O. Blanke, First person experience of body transfer in virtual reality, PloS one, 2010, Vol. 5(5), pp. e10564.

[19] J. Heyse, F. Ongenae, J. De Letter, A. All, F. De Backere, F. De Turck, Design of an ontology for decision support in VR exposure therapy, 13th EAI International Conference on Pervasive Computing Technologies for Healthcare, Trento, 2019.

[20] J. MacQueen, Some methods for classification and analysis of multivariate observations, Proceedings of the fifth Berkeley symposium on mathematical statistics and probability, 1967, Vol. 1(14), pp. 281297.

[21] F.C. Hsu, A.J.C. Trappey, C.V. Trappey, J.L. Hou, S.F. Liu, Technology and knowledge document cluster analysis for enterprise $\mathrm{R} \& \mathrm{D}$ strategic planning. International Journal of Technology Management, 2006, Vol. 36(4), pp. 336-353.

[22] A.J.C. Trappey, P.P. Chen, C.V. Trappey, L. Ma, A machine learning approach for solar power technology review and patent evolution analysis. Applied Sciences, 2019, Vol. 9(7), 1478.

[23] A. C. Jhuang, J. J. Sun, A.J.C. Trappey, C.V. Trappey, U. H. Govindarajan, Computer supported technology function matrix construction for patent data analytics. IEEE 21st International Conference on Computer Supported Cooperative Work in Design (CSCWD), Wellington, 2017, pp. 457-462. 